\title{
Physiotherapy plus conventional treatment versus conventional treatment only in the treatment of functional constipation in children: design of a randomized controlled trial and cost-effectiveness study in primary care
}

Jojanneke J. G. T. van Summeren '10, Gea A. Holtman', Yvonne Lisman- van Leeuwen', Lisa E. A. M. Louer', Alice H. C. van Ulsen-Rust ${ }^{2}$, Karin M. Vermeulen ${ }^{3}$, Boudewijn J. Kollen ${ }^{1}$, Janny H. Dekker ${ }^{1}$ and Marjolein Y. Berger ${ }^{{ }^{*}}$

\begin{abstract}
Background: Our aim was to design a study to evaluate the effectiveness and cost-effectiveness of adding physiotherapy to conventional treatment for children with functional constipation in primary care. Physiotherapy is focusing on improving the coordination between the pelvic floor and abdominal musculature during bowel movement, while conventional treatment is mainly focusing on symptomatic relief of symptoms, therefore, we expect the effects of physiotherapy will be more sustainable than the effects of conventional treatment. In this paper we describe the final study design and how the design was adapted, to overcome recruitment problems.

Methods: We designed a randomized controlled trial of children aged 4-17 years with functional constipation diagnosed by a general practitioner or pediatrician. Children in the intervention group received physiotherapy plus conventional treatment, and those in the control group received conventional treatment only. Follow-up measurements took place at 4 and 8 months. The primary outcome was treatment success defined according to the Rome-III criteria as the absence of functional constipation, with no laxative use. Secondary outcomes were absence of functional constipation irrespective of laxative use, quality of life, global perceived effect, and costs. Children were recruited from September 2014 to February 2017. Initially, we aimed to include children with recent symptom onset. However, in the first phase of enrollment we were confronted with an unforeseen recruitment problem: many children and their parents refused randomization because physiotherapy was considered too burdensome for the stage of disease. Therefore, we decided to also include children with a longer duration of symptoms. In total 134 children were included.
\end{abstract}

Discussion: The target number of participants is achieved. Therefore, the results may change thinking about the management of functional constipation in children.

Trail registration: Netherlands Trial Register (NTR4797), registered 8 September 2014.

Keywords: Constipation, General practitioner, Pelvic floor, Child and adolescent, Family medicine

\footnotetext{
* Correspondence: m.y.berger@umcg.nl

${ }^{1}$ Department of General Practice and Elderly Care Medicine, University of

Groningen, University Medical Center Groningen, Groningen, The

Netherlands

Full list of author information is available at the end of the article
}

(c) The Author(s). 2018 Open Access This article is distributed under the terms of the Creative Commons Attribution 4.0 International License (http://creativecommons.org/licenses/by/4.0/), which permits unrestricted use, distribution, and reproduction in any medium, provided you give appropriate credit to the original author(s) and the source, provide a link to the Creative Commons license, and indicate if changes were made. The Creative Commons Public Domain Dedication waiver (http://creativecommons.org/publicdomain/zero/1.0/) applies to the data made available in this article, unless otherwise stated. 


\section{Background}

Functional constipation (FC) is a common problem in children [1]. Its etiology is multifactorial, involving age, behavior, pelvic floor function, and gastrointestinal motility. Conventional treatment includes education, dietary advice, toilet training, and laxatives [2, 3]. However, despite this multifaceted approach, $50 \%$ of children still experience FC after 6-12 months' treatment with laxatives and $25 \%$ have symptoms that persist into adulthood $[4,5]$. Therewith FC has not only a major impact on the quality of life of both children and their families but also increases healthcare costs significantly [6-8].

The pelvic floor and abdominal muscles contract and relax in a coordinated manner during bowel movements, and dysfunction of this interaction could be important in the onset and maintenance of FC [9]. Children with FC, either consciously or unconsciously, appear to strain their pelvic floor muscles and fail to relax the external anal sphincter during bowel movements [10, 11]. In addition, reduced trunk stability may preclude achievement of the posture and intra-abdominal pressure required for defecation $[10,12]$. Physiotherapy for FC focuses on improving this coordination between the abdominal and pelvic floor musculature [9]. To date, two small clinical trials in specialist care have shown promising results for the effects of physiotherapy in children with FC $[13,14]$. Physiotherapy is expected to give optimal results in children with recent symptom onset [4]. Therefore, it could be particularly effective in children presenting to primary care.

We aimed to design a randomized controlled trial to evaluate the effectiveness and cost-effectiveness of physiotherapy plus conventional treatment in comparison to conventional treatment alone for children aged 4-17 years presenting with FC in primary care. We encountered problems in the recruitment of participants that led us to change the original criteria for participant selection. In this paper we therefore start with presenting our final study design. Thereafter, we describe the process of recruiting participants, including the changes implemented in the original study design. Lastly, we evaluate the representativeness of our study population by comparing characteristics of children that participated and refused to participate in this trial.

\section{Methods}

\section{Design}

We designed a randomized controlled trial that had a follow-up period of 8 months, and wherein children were included between September 2014 and March 2017. The trial was approved by the Medical Ethical Board of the University Medical Center of Groningen (METC2013/331) and was registered in the Netherlands Trial Register (NTR4797). Before enrollment we obtained written informed consent from all parent(s). In addition, children aged $\geq 12$ years provided written informed consent themselves.

\section{Participants \\ Eligibility criteria}

Children were eligible for inclusion if aged 4-17 years and diagnosed with FC by a general practitioner (GP) or general pediatrician. Specifically, children were required to have experienced FC symptoms or to have used laxatives in the 4 weeks before enrollment. Children under the age of 4 years were considered too young to undergo physiotherapy [9]. The exclusion criteria were psychopathology affecting protocol adherence, severe disease (physician determined), and physiotherapy or urotherapy for constipation in the past 3 years (Fig. 1).

\section{Patient recruitment}

We recruited all children (aged 4-17 years) presenting to general practices or who were newly referred to pediatric outpatient departments with a diagnosis of FC. During the first consultation for FC symptoms, parents and children were informed about this study by their treating physician (incident cases). In addition, any children with a known diagnosis of FC and who had consulted their GP in the past 12 months for this diagnosis were sent a leaflet explaining the study (prevalent cases). Children or their parents (depending on the child's age) were asked to complete a short questionnaire supplied with the leaflet, detailing whether the child had experienced symptoms of FC or used laxatives in the past 4 weeks. Once completed, they were asked to return the questionnaire.

\section{Interventions}

\section{Control group: conventional treatment only}

Children in the control group received conventional treatment. No restrictions or recommendations were given to the physicians regarding treatment. However, education, dietary advice, toilet training, and when indicated, laxative prescribing were permitted based on appropriate guidance $[2,3]$.

\section{Intervention group: physiotherapy plus conventional treatment}

Children in the intervention group received physiotherapy in addition to conventional treatment. Physiotherapy consisted of a maximum of nine half-hour sessions carried out by specialist physiotherapists, and ended if the physiotherapist considered that treatment was successful or that no more improvement was expected. The physiotherapists were trained to master's degree level in pediatric or pelvic physiotherapy, and had received postgraduate education in the treatment of bladder and 


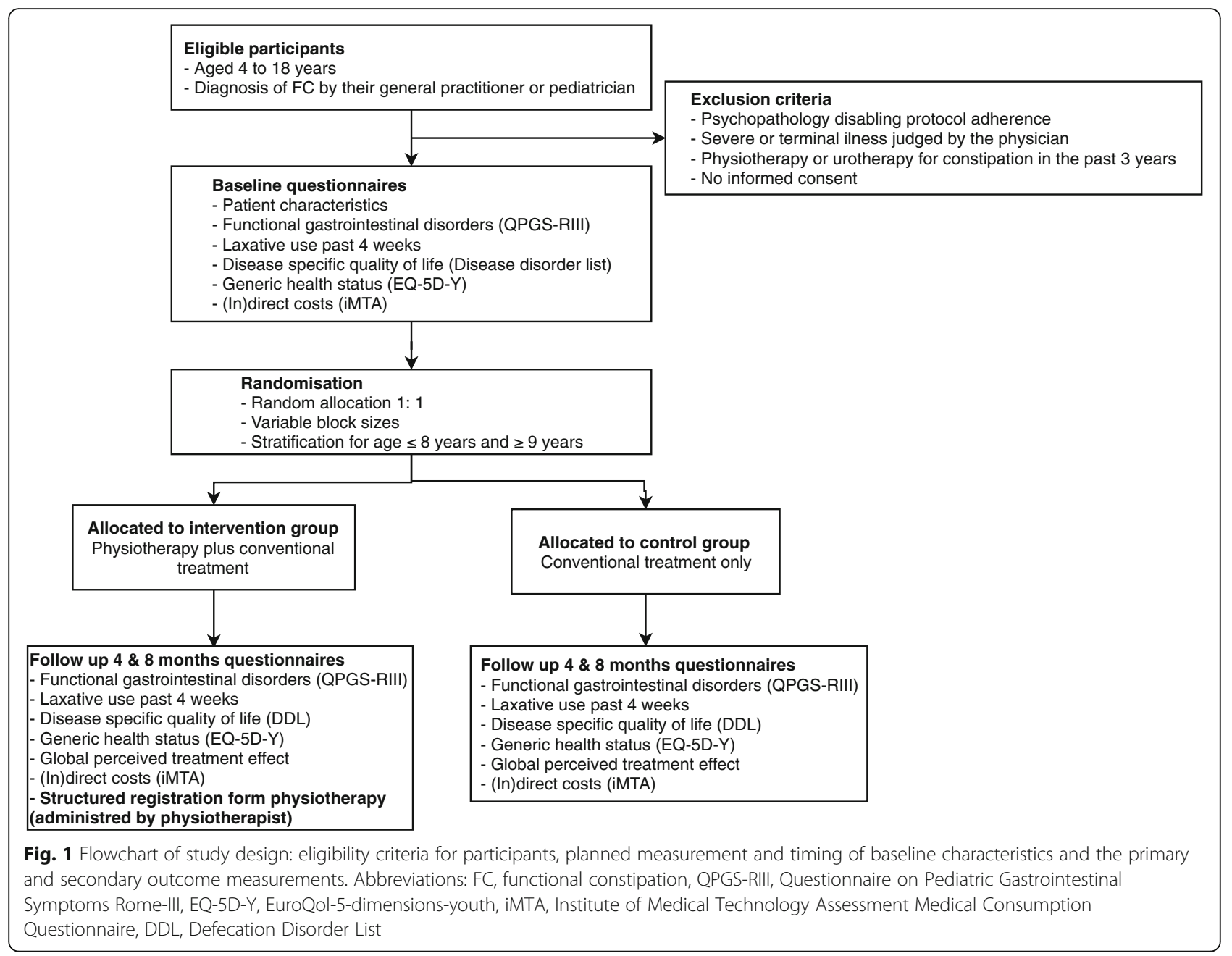

bowel dysfunction in children. The patient-tailored structured treatment program used in this study was developed in cooperation with experienced specialist physiotherapists and approved by all participating specialist physiotherapists before the study. The physiotherapist tailored the treatment program to the individual patient. For each patient and session, the physiotherapists recorded on a structured form the treatment options used to reach the six goals.

In young children, physiotherapy focused on the child and his or her parent(s), while in older children, the focus was mainly on the child. All exercises, materials, and methods were presented in a manner appropriate to the child's developmental age and locomotor skills. For the patient-tailored structured treatment program used in this study, the six goals were: 1 ) improving the knowledge about defecation, and the role that the child and/ or parent might have in the persistence of symptoms; 2 ) improving toileting behavior and practicing a stable toilet posture; 3) increasing awareness of the sensation of needing to defecate; 4 ) learning to relax while defecating;
5) learning to breath correctly to generate adequate intra-abdominal pressure for defecation; and 6) teaching effective straining for defecation. Biofeedback and electrotherapy were not allowed in this study because there is insufficient evidence of their efficacy in children with FC and because we considered these therapies too invasive for treatment of children presenting to primary care [15].

\section{Randomization and blinding}

Children were randomly allocated in a 1:1 ratio to the two treatment groups, using a computer-generated randomization list with random block sizes. Randomization was stratified into those aged 4-8 years and those aged 9-17 years. The randomization list was maintained by a researcher who was not involved in the study and had no access to the allocation site.

Children, parents, physicians, and physiotherapists could not be blinded to the intervention. The investigator was blinded to the assigned study group during data entry and statistical analyses. 


\section{Outcomes}

The primary outcome was treatment success, defined according to the Rome-III criteria as the absence of FC without laxative use (see Table 1 for the Rome-III criteria used to define FC) [16]. Thus, a successfully treated child was required to fulfill none or one of the six Rome-III criteria. Other secondary outcomes were absence of FC according to the Rome-III criteria irrespective of laxative use, quality of life, global perceived treatment effect, and costs.

\section{Measurements}

Figure 1 gives an overview of the measurement and timing of baseline characteristics and the primary and secondary outcome parameters; follow-up measurements took place after 4 and 8 months. We collected the following data at baseline: age, gender, symptom duration, age at symptom onset, symptom chronicity, and whether lower urinary tract symptoms were present. Symptom chronicity was defined as continuous or regular laxative use ( $\geq 3$ periods) in the 12 months before inclusion.

\section{Measurement of the primary outcome}

The presence of FC was assessed with a Dutch version of the Questionnaire on Pediatric Gastrointestinal Symptoms Rome-III (QPGS-RIII) [16]. This standardized questionnaire was used to assess if children have experienced functional gastrointestinal symptoms over the last 2 months. We adapted the questionnaire and evaluated symptoms over a period of 4 weeks. Children completed the questionnaire themselves if they were aged 13-17 years, but parents completed the questionnaire if their child was aged 4-12 years. In addition, parents answered the question "Did your child use laxatives in the past four weeks?" (yes or no). If one or more responses were missing for the primary outcome measure, we contacted the child or parent by telephone to obtain an answer.

Table 1 Description of the Rome III criteria for functional constipation [16]

According to the ROME III criteria, a child must have a developmental age of at least 4 years and fulfill two or more of the following criteria, at least two months prior to diagnosis ${ }^{\mathrm{a}}$ :

1) two or fewer defecations in the toilet per week,

2) at least one episode of fecal incontinence per week,

3) history of retentive posturing or excessive volitional stool retention at least once a week,

4) history of painful or hard bowel movement at least once a week,

5) presence of a large fecal mass in the rectum at least once a week,

6) history of large diameter stools that may obstruct the toilet at least once a week.

${ }^{a}$ For the purpose of this study, patients were eligible for enrollment if symptoms were present for at least one month before diagnosis, rather than two months, which is in agreement with the recently published Rome-IV criteria [27]

\section{Measurement of secondary outcomes}

Disease-specific quality of life was measured with the Defecation Disorder List (DDL), [17, 18] adapted to include only the emotional and social functioning subdomains. The constipation-related and treatment/ intervention subdomains were omitted because it has previously been demonstrated that these have low internal consistency and potentially low validity when used to measure disease-specific quality of life $[17,18]$. Health status was measured with the EuroQol-5-dimensions-youth (EQ-5D-Y) [19]. Proxy report versions of the DDL and EQ5D-Y questionnaires were completed by parents, and children aged $\geq 8$ years also completed child self-reports. The global perceived treatment effect of patients (GPE) was scored by parents and measured with a 9-point Likert-type scale (very much, much, reasonable, and slightly improved; no change; slightly, reasonable, much, and very much worse). When parents reported that the symptoms of their child were improved very much or much we defined the treatment as successful.

Healthcare consumption related to FC, such as GP or pediatrician visits, drug treatment, and parental productivity loss, were measured with versions of the Institute of Medical Technology Assessment Medical Consumption Questionnaire (iMTA-MCQ) and the Productivity Costs Questionnaire (iMTA-PCQ), respectively, adjusted for FC $[20,21]$. Both cost questionnaires were completed by parents only.

If questionnaires were not returned, participants were sent a reminder e-mail after 2 weeks and received a reminder telephone call after 3 weeks.

\section{Sample size}

Sample size estimates were based on a systematic literature review showing that after 6 to 12 months of conventional treatment, $50 \%$ of the children were free of symptoms without using laxatives [22]. Physiotherapy in one study has been shown to improve outcomes by $30 \%$ compared with conventional treatment alone in children with FC referred to pediatric specialist care [14]. However, that study may have overestimated the magnitude of effect because it was small and underpowered [23]. Therefore, we used a more conservative estimate of the difference in treatment success (25\%) between the intervention and control group. The sample size was calculated with expected treatment success rates after 6-12 months of 50 and $75 \%$ in the conventional and intervention groups, respectively [14, 22]. Given an expected loss to follow-up of $10 \%$, we estimated a total sample size of 128 children (alpha 0.05 , power 0.80 ).

\section{Statistical analyses}

We will use appropriate descriptive statistics to describe patient characteristics, baseline questionnaire scores, 
and the proportions of successfully treated children at 4 and 8 months in the intervention and control groups.

We will use multilevel analyses to investigate the longitudinal relationship between the intervention group (physiotherapy plus conventional treatment) and the control group (conventional treatment) with respect to the primary and secondary outcome variables. The applied levels will be repeated measures (that is, time), and patient. We will base our analyses on intention to treat (ITT). The ITT population will consist of all patients who have given informed consent and have been randomly allocated to one of the two treatments, irrespective of whether they received the allocated treatment or not. An additional secondary per protocol analysis (PP) will be conducted for the outcome variable. The PP population will consist of all children randomized in the intervention group receiving at least one physiotherapy session and all children in the control group that had no physiotherapy. Finally, in a sensitivity analysis we will evaluate whether the effect of the intervention is different for children with and without chronic symptoms.

\section{Economic evaluation}

A cost-effectiveness analysis is planned. The primary aim will be to estimate the societal costs, and the secondary aim will be to estimate the cost-effectiveness of treatment with physiotherapy plus conventional treatment compared to conventional treatment alone. In addition, we will perform a cost-utility analysis based on EuroQol-defined utilities. The parental version of the EQ5D-Y questionnaire will be used for these evaluations. The cost-effectiveness analyses will then be expressed as incremental cost-effectiveness ratios (ICERs), displaying the extra treatment costs of physiotherapy to gain one extra patient with successful treatment, as compared with conventional care. In turn, cost-utility analyses will be expressed as incremental cost-utility ratios (ICURs), displaying the extra costs to gain one additional quality-adjusted life year (QALY). Given that the study follow-up was only planned to be 8 months, we will not include discounting of costs and effects. Bootstrap resampling will be performed on the cost and effect pairs to estimate confidence intervals more accurately and to create a cost-effectiveness plane.

\section{Process evaluation of adaptations to the original study design \\ Criteria for participant eligibility}

We had intended to include only those children with FC of recent onset. Therefore, we originally excluded children who were using laxatives or who had used laxatives in the previous 3 months. However, when study enrollment started in September 2014, we were confronted unexpectedly with the fact that many children and parents refused to participate in this trial because they considered the symptoms were not severe enough to justify referral for physiotherapy, which could occur if they consented in randomization. Consequently, many of these patients preferred to opt for laxatives before considering referral to physiotherapy. After recruiting only 20 children over a 12-month period, we decided to expand our eligibility criteria to include also those children who were currently using, or who had used, laxatives in the previous 3 months. This meant that our study population was expanded with children with more advanced FC. Expanding the inclusion criteria also allowed us to include children who had been seen by their GP for FC in the past 12 months, as well as consecutive children newly referred to pediatric outpatient departments. For budgetary reasons, the delay in participant recruitment forced us to shorten the planned follow-up period from 12 months to 8 months. The Medical Ethical Board of the University Medical Center of Groningen approved these changes in study design (METC2013/331).

\section{Sample size calculation}

The original sample size calculation was based on conventional treatment being successful in $60 \%$ of children consulting their GP for the first time for FC [24]. At that time, no studies had reported on the treatment effects of physiotherapy, and we estimated a $20 \%$ difference in treatment success between the intervention and control groups to be relevant [22]. Thus, we expected the treatment under study would be successful in $80 \%$ of the children receiving physiotherapy. Given an expected loss to follow-up of $10 \%$, we had calculated that 180 children would be required for the study (alpha 0.05 , power 0.80 ). However, since the original design, a study had been reported on the effectiveness of physiotherapy in childhood FC in a pediatric outpatient department [14]. Therefore, coupled with the changes in study design, we reconsidered our sample size calculation (see methods section).

\section{Representativeness of the finally selected study population}

Children were recruited from 93 general practices (209 GPs) and 5 general pediatric outpatient departments in district hospitals between September 2014 and March 2017. Of the 224 children assessed for eligibility, 213 children were invited by GPs: 44 children with a new diagnosis (incident cases), and 169 children with a diagnosis of FC within the past 12 months (prevalent cases); and 11 newly referred children were invited by pediatricians (Fig. 2).

We compared patient characteristics between children included in the trial (participants, $n=134$ ) and children who refused to participate or who met the exclusion criteria (non-participants, $n=90$ ) (Fig. 2 and Table 2). 


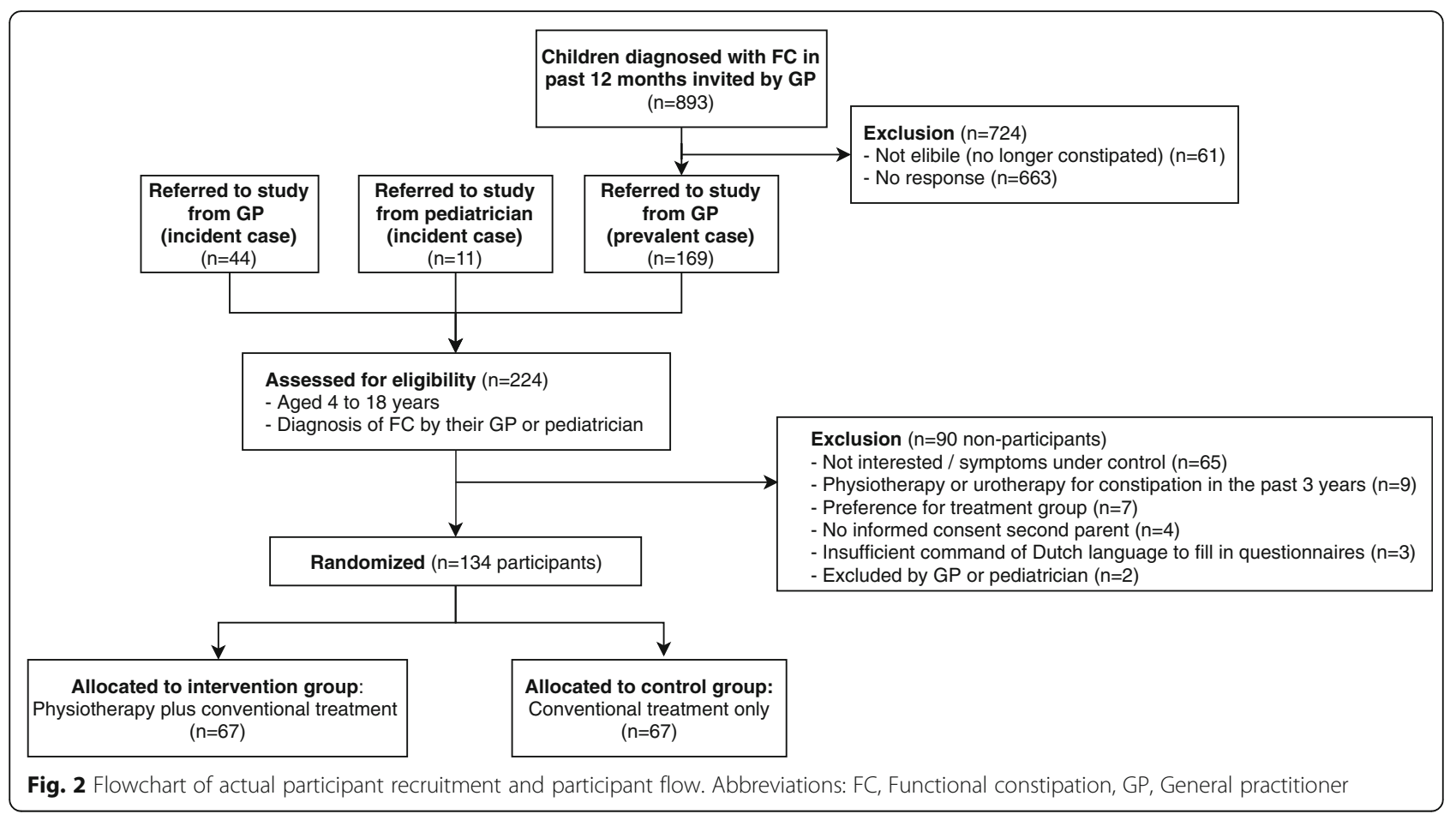

Participants were found to be slightly younger (mean age, $7.5 \pm 3.5$ years) compared with non-participants (mean age, $8.2 \pm 3.8$ years), but the boy-to-girl ratio was comparable (Table 2). Among non-participants, symptom chronicity was only assessed in children referred as prevalent cases by their GP for logistical reasons. Comparing chronicity among prevalent cases seems to show that participants more often had chronic symptoms at baseline (65\%) compared with non-participants (43\%) (Table 2).

\section{Discussion}

Although two small clinical trials have shown that physiotherapy for FC could be a promising treatment for children in specialist care, $[13,14]$ we are not aware of any trial evaluating its effectiveness in primary care where most children with FC are diagnosed and treated [25]. The aim of physiotherapy is to improve the coordination between the abdominal and pelvic floor musculature during bowel movement [9]. The strength of physiotherapy is that physical exercises are combined with cognitive and behavioral elements, such as education and toilet training [26]. Treatment guidelines recommend that these cognitive and behavioral elements be discussed by doctors during a consultation [2, 3] . However, this might be problematic because GPs focus on symptomatic relief of symptoms. In addition, the

Table 2 Characteristics of participants and non-participants

\begin{tabular}{|c|c|c|}
\hline & Participants $(N=134)$ & Non-participants $(N=90)$ \\
\hline Age (mean, SD) & $7.5 \pm 3.46$ & $8.23 \pm 3.80^{a}$ \\
\hline Gender (\% girls) & 61.2 & $60.0^{\mathrm{a}}$ \\
\hline \multicolumn{3}{|l|}{ Referred to study by: } \\
\hline - GP (incident case), (n, \%) & $22(17 \%)$ & $22(24 \%)$ \\
\hline - Pediatrician (incident case), (n, \%) & $6(4 \%)$ & $5(6 \%)$ \\
\hline - GP (prevalent case), ( $(n, \%)$ & $106(79 \%)$ & $63(70 \%)$ \\
\hline \multicolumn{3}{|c|}{ Chronicity of symptoms before randomization b, c } \\
\hline -Yes (n, \%) & $67(65 \%)$ & $16(43 \%)$ \\
\hline$-\mathrm{No}(\mathrm{n}, \%)$ & $36(35 \%)$ & $21(57 \%)$ \\
\hline
\end{tabular}

GP General practitioner, SD standard deviation

${ }^{a}$ Age and gender were not available of 19 non-participants

${ }^{b}$ Comparison of chronicity of symptoms between participants and non-participants, was only performed for prevalent cases in whom the question about chronicity was asked (participants $n=103$, non-participants $n=37$ )

c Symptom chronicity was defined as continuous or regular laxative use ( $\geq 3$ periods) in the 12 months before inclusion 
consultation time is only $10 \mathrm{~min}$ in primary care, which limits the time for proper education, and advices on toilet training.

Initially, we had aimed to assess physiotherapy in children with recent-onset FC, for two main reasons. First, we assume that the long-term prognosis could be more improved if children receive treatment early in the disease process [4]. Second, we think that duration of symptoms and of treatments would be more homogenous in children with recent onset of symptoms. However, we discovered that parents and children could not be motivated for a time-intensive therapy like physiotherapy for symptoms they considered to be temporary and mild. Our subsequent comparison of participants and non-participants confirmed that children were more inclined to participate if they had longer symptom durations and regular laxative use. Therefore, our study will generate results on the effects of physiotherapy for children with more advanced FC than we had originally planned. Specifically, we expect our results to concern those cases where the child or parent have experienced conventional primary care treatment to be insufficient.

We hypothesized that physiotherapy, by increasing awareness of the abdominal and pelvic floor muscle use during defecation, would have a more sustained effect on outcomes than symptomatic treatment with laxatives. Although we were therefore particularly interested in the long-term effects of physiotherapy, the follow-up duration had to be shortened from 12 to 8 months. However, a follow-up duration of 12 months is probably also too short to evaluate whether the effects of physiotherapy are sustainable. The results of this study will help deciding if it is justified to plan a long term follow-up study.

\section{Clinical impact}

We designed the first trial to evaluate the effectiveness of physiotherapy as a first-line treatment for childhood FC in primary care. In total 134 children were included, giving this study sufficient power to lead to promising results. These results may change thinking about the management of functional constipation in children.

\section{Abbreviations \\ DDL: Defecation disorder list; EQ5D-Y: EuroQol-5-dimensions-youth; FC: Functional constipation; GP: General practitioner; GPE: Global perceived effect; ICER: Incremental cost-effectiveness ratio; ICUR: Incremental cost-utility ratio; iMTA-MCQ: Institute of Medical Technology Assessment Medical Consumption Questionnaire; iMTA-PCQ: Institute of Medical Technology Assessment Productivity Costs Questionnaire; ITT: Intention to treat; PP: Per protocol; QALY: Quality-adjusted life year; QPGS-RIII: Questionnaire on Pediatric Gastrointestinal Symptoms Rome-III}

\section{Acknowledgments}

We would like to thank the primary care physicians and general pediatricians for recruiting and treating the patients in this study. We would also like to thank the physiotherapists who assisted in the development of the patienttailored treatment program, as well as the physiotherapists who performed the physiotherapy interventions. The editorial assistance by Dr. Robert Sykes (http://www.doctored.org.uk) was appreciated. Last but not least, we are grateful to all participants for their contributions.

\section{Funding}

This trial is funded by the Netherlands organization for Health Research and Development (ZonMw), project number 837001409 . The funding organization had no role in the design or conduct of the study; the collection, management, analysis, or interpretation of the data; the preparation, review, or approval of the manuscript; or the decision to submit the manuscript for publication.

\section{Availability of data and materials}

The datasets used and/or analysed during the current study are available from the corresponding author on reasonable request.

\section{Authors' contributions}

All authors were responsible for the reported research, have participated in the concept and design, and have approved the manuscript as submitted. JVS and GH drafted the initial version of the manuscript. BK, JD, and MB critically reviewed and revised the initial version of the manuscript. $Y L V L, L L$, AUR and KV critically reviewed the final version of the manuscript.

\section{Ethics approval and consent to participate}

The trial was approved by the Medical Ethical Board of the University Medical Center of Groningen (METC2013/331). We obtained informed consent from both the parent(s) and the child (if aged $\geq 12$ years) before enrollment.

\section{Consent for publication}

Not applicable.

\section{Competing interests}

The authors declare that they have no competing interests.

\section{Publisher's Note}

Springer Nature remains neutral with regard to jurisdictional claims in published maps and institutional affiliations.

\section{Author details}

${ }^{1}$ Department of General Practice and Elderly Care Medicine, University of Groningen, University Medical Center Groningen, Groningen, The Netherlands. ${ }^{2}$ Pelvic Physiotherapy, Practices for Pelvic Physiotherapy, Groningen, The Netherlands. ${ }^{3}$ Department of Epidemiology, University of Groningen, University Medical Center Groningen, Groningen, the Netherlands.

Received: 1 March 2018 Accepted: 19 July 2018

Published online: 31 July 2018

\section{References}

1. Mugie SM, Di Lorenzo C, Benninga MA. Constipation in childhood. Nat Rev Gastroenterol Hepatol. 2011;8(9):502-11.

2. Nederlandse Vereniging voor Kindergeneeskunde, Nederlands Huisarts Genootschap: Richtlijn obstipatie bij kinderen van 0 tot 18 jaar. 2009. http:// www.nvk.n//Portals/0/richtlijnen/Obstipatie/Obstipatie\%20bij\%20kinderen \%20van\%200\%20tot\%2018\%20jaar\%20revisie\%202016.pdf

3. National Collaborating Centre for Women's and Children's Health (UK): Constipation in children and youn people: diagnosis and management of idiopathic childhood constipation in primary and secondary care. 2010, https://www.nice.org.uk/guidance/cg99.

4. Bongers ME, van Wijk MP, Reitsma JB, Benninga MA. Long-term prognosis for childhood constipation: clinical outcomes in adulthood. Pediatrics. 2010; 126(1):e156-62.

5. Pijpers MA, Bongers ME, Benninga MA, Berger MY. Functional constipation in children: a systematic review on prognosis and predictive factors. J Pediatr Gastroenterol Nutr. 2010;50(3):256-68.

6. Belsey J, Greenfield S, Candy D, Geraint M. Systematic review: impact of constipation on quality of life in adults and children. Aliment Pharmaco Ther. 2010;31(9):938-49. 
7. Liem O, Harman J, Benninga M, Kelleher K, Mousa H, Di Lorenzo C. Health utilization and cost impact of childhood constipation in the United States. J Pediatr. 2009;154(2):258-62.

8. Choung RS, Shah ND, Chitkara D, Branda ME, Van Tilburg MA, Whitehead $W E$, et al. Direct medical costs of constipation from childhood to early adulthood: a population-based birth cohort study. J Pediatr Gastroenterol Nutr. 2011;52(1):47-54.

9. van Engelenburg-van Lonkhuyzen ML, Bols EM, Benninga M, Groen L, Chase J, de Bie R. Physiotherapy interventions for functional bladder and bowel dysfunctions in neurologically normal and otherwise healthy children. Cochrane Library. 2016;

10. Loening-Baucke V, Cruikshank B, Savage C. Defecation dynamics and behavior profiles in encopretic children. Pediatrics. 1987;80(5):672-9.

11. van Engelenburg-van Lonkhuyzen ML, Bols EM, Benninga MA, Verwijs WA, de Bie RA. Bladder and bowel dysfunctions in 1748 children referred to pelvic physiotherapy: clinical characteristics and locomotor problems in primary, secondary, and tertiary healthcare settings. Eur J Pediatr. 2017; 176(2):207-16

12. Chase JW, Stillman BC, Gibb SM, Clarke MC, Robertson VJ, Catto-Smith AG, et al. Trunk strength and mobility changes in children with slow transit constipation. J Gastroenterol Hepatol. 2009;24(12):1876-84.

13. Silva C, Motta M. The use of abdominal muscle training, breathing exercises and abdominal massage to treat paediatric chronic functional constipation. Color Dis. 2013;15(5):e250-5.

14. van Engelenburg-van Lonkhuyzen ML, Bols EM, Benninga MA, Verwijs WA, de Bie RA. Effectiveness of pelvic physiotherapy in children with functional constipation compared with standard medical care. Gastroenterology. 2017; 152(1):82-91.

15. Brazzelli M, Griffiths PV, Cody JD, Tappin D. Behavioural and cognitive interventions with or without other treatments for the management of faecal incontinence in children. Cochrane Library. 2011;

16. Rasquin A, Di Lorenzo C, Forbes D, Guiraldes E, Hyams JS, Staiano A, et al. Childhood functional gastrointestinal disorders: child/adolescent. Gastroenterology. 2006;130(5):1527-37.

17. Voskuijl WP, van der Zaag-Loonen HJ, Ketel IJ, Grootenhuis MA, Derkx BH, Benninga MA. Health related quality of life in disorders of defecation: the defecation disorder list. Arch Dis Child. 2004:89(12):1124-7.

18. Bongers ME, van Dijk M, Benninga MA, Grootenhuis MA. Health related quality of life in children with constipation-associated fecal incontinence. J Pediatr. 2009;154(5):749-53. e1

19. Wille N, Badia X, Bonsel G, Burström K, Cavrini G, Devlin N, et al. Development of the EQ-5D-Y: a child-friendly version of the EQ-5D. Qual Life Res. 2010;19(6):875-86.

20. Bouwmans C, Hakkaart-van Roijen L, Koopmanschap M, Krol M, Severens H, Brouwer W. Handleiding iMTA Productivity Cost Questionnaire (iPCQ). Rotterdam: iMTA, Erasmus Universiteit; 2013.

21. Bouwmans C, Hakkaart-van Roijen L, Koopmanschap MA, Krol M, Severens $J$, Brouwer W. IMTA medical consumption questionnaire: handleiding: Institute for Medical Technology Assessment, Erasmus Universiteit Rotterdam; 2013.

22. Pijpers MA, Tabbers MM, Benninga MA, Berger MY. Currently recommended treatments of childhood constipation are not evidence based: a systematic literature review on the effect of laxative treatment and dietary measures. Arch Dis Child. 2009;94(2):117-31.

23. van Summeren J, Dekker J, Berger M. Pelvic physiotherapy in children with functional constipation: promising but more research needed. Gastroenterology. 2017;152(8):2080-1.

24. Borowitz SM, Cox DJ, Kovatchev B, Ritterband LM, Sheen J, Sutphen J. Treatment of childhood constipation by primary care physicians: efficacy and predictors of outcome. Pediatrics. 2005;115(4):873-7.

25. Burgers R, Bonanno E, Madarena E, Graziano F, Pensabene L, Gardner W, et al. The care of constipated children in primary care in different countries. Acta Paediatr. 2012;101(6):677-80,

26. Frawley HC, Dean SG, Slade SC, Hay-Smith EJC. Is pelvic-floor muscle training a physical therapy or a behavioral therapy? A call to name and report the physical, cognitive, and behavioral elements. Phys Ther. 2017;97(4):425-37.

27. Hyams JS, Di Lorenzo C, Saps M, Shulman RJ, Staiano A, van Tilburg M. Childhood functional gastrointestinal disorders: child/adolescent. Gastroenterology. 2016;150(6):1456-68. e2

Ready to submit your research? Choose BMC and benefit from:

- fast, convenient online submission

- thorough peer review by experienced researchers in your field

- rapid publication on acceptance

- support for research data, including large and complex data types

- gold Open Access which fosters wider collaboration and increased citations

- maximum visibility for your research: over $100 \mathrm{M}$ website views per year

At BMC, research is always in progress.

Learn more biomedcentral.com/submissions 